\title{
1-乙基-3-甲基咪唑丙氨酸离子液体热力学性质的研究
}

\author{
郑玲 ${ }^{1}$ 樊本汉 ${ }^{1}$ 卜晓雪 ${ }^{1}$ 潘 懿 ${ }^{1}$ 董家新 ${ }^{2}$ 关 伟 ${ }^{1, *}$ \\ ('辽宁大学化学院, 沈阳 110036; 2 广西师范大学化学化工学院, 广西 桂林 541004)
}

\begin{abstract}
摘要: 用中和法合成了氨基酸离子液体1-乙基-3-甲基咪唑丙氨酸 $\left[\mathrm{C}_{2} \mathrm{mim}\right][\mathrm{Ala}]$ ), 并利用恒温环境的溶解反应 热量计, 在 $(288.15 \pm 0.01) \mathrm{K}-(308.15 \pm 0.01) \mathrm{K}$ 温度范围内每隔 $5 \mathrm{~K}$, 测定不同质量摩尔浓度离子液体在水中 的溶解焓 $\left(\Delta_{\mathrm{sol}} H_{\mathrm{m}}\right)$. 根据Archer的方法, 通过线性拟合得到了该离子液体的标准摩尔溶解焓 $\left(\Delta_{\mathrm{sol}} H_{\mathrm{m}}^{\theta}\right)$, 并计算了其 相对表观摩尔溶解焓 $\left({ }^{\Phi} L\right)$. 在 $298.15 \mathrm{~K}$, 根据Glasser经验方法得到了格子能 $U_{\mathrm{POT}}=566 \mathrm{~kJ} \cdot \mathrm{mol}^{-1}$, 并计算了 其阴阳离子水化焓值 $\left(\Delta H^{+}+\Delta H\right)=-620 \mathrm{~kJ} \cdot \mathrm{mol}^{-1}$ 及阴离子水化焓 $\Delta H\left([\mathrm{Ala}]^{-}\right)=-387 \mathrm{~kJ} \cdot \mathrm{mol}^{-1}$. 此外, 估算了 $\left[\mathrm{C}_{2} \mathrm{mim}\right][\mathrm{Ala}]$ 水溶液的热容 $\left(C_{p(\mathrm{sol})}\right)$ 和表观摩尔热容 $\left({ }^{\Phi} C_{p}\right)$.
\end{abstract}

关键词: 离子液体; 溶解焓; 热容; 水化焓; 格子能

中图分类号: 0642

\section{Study on Thermodynamic Properties of Ionic Liquid 1-Ethyl-3-methylimidazolium Alanine}

\author{
ZHENG Ling ${ }^{1} \quad$ FAN Ben-Han ${ }^{1} \quad$ BU Xiao-Xue ${ }^{1} \quad$ PAN Yi ${ }^{1} \quad$ DONG Jia-Xin ${ }^{2} \quad$ GUAN Wei ${ }^{1, *}$ \\ ('College of Chemistry, Liaoning University, Shenyang 110036, P. R. China; ${ }^{2}$ College of Chemistry \& Chemical Engineering, \\ Guangxi Normal University, Guilin 541004, Guangxi Province, P. R. China)
}

\begin{abstract}
We synthesized the alanine-based ionic liquid [ $\mathrm{C}_{2}$ mim][Ala] (1-ethyl-3-methylimidazolium alanine) by using the neutralization method and characterized it. Using a solution-reaction isoperibol calorimeter, we determined the molar enthalpies of the solution $\left(\Delta_{\mathrm{sol}} H_{\mathrm{m}}\right)$ at various molalities in water from $(288.15 \pm 0.01)$ to $(308.15 \pm 0.01) \mathrm{K}$ in intervals of $5 \mathrm{~K}$. Using Archer's method, we obtained the standard molar enthalpy of solution for [ $\mathrm{C}_{2}$ mim] [Ala] $\left(\Delta_{\mathrm{sol}} H_{\mathrm{m}}^{\theta}\right)$ and calculated its apparent relative molar enthalpy $\left({ }^{\Phi} \mathrm{L}\right)$. Using Glasser's theory of lattice energy, we obtained the lattice energy, $U_{\text {РОT }}=566 \mathrm{~kJ} \cdot \mathrm{mol}^{-1}$, the hydration enthalpy of the cation and anion, $\left(\Delta H^{+}+\Delta H\right)=-620 \mathrm{~kJ} \cdot \mathrm{mol}^{-1}$, and the hydration enthalpy of an anion, $\Delta H\left([\text { Ala] }]^{-}\right)=-387$ $\mathrm{kJ} \cdot \mathrm{mol}^{-1}$ at $298.15 \mathrm{~K}$. Finally, we obtained the heat capacity of aqueous $\left[\mathrm{C}_{2} \mathrm{mim}\right][\mathrm{Ala}]\left(C_{p(\mathrm{sol})}\right)$ and its apparent molar heat capacity $\left({ }^{\oplus} C_{p}\right)$ at various specific molalities.
\end{abstract}

Key Words: Ionic liquid; Enthalpy of solution; Heat capacity; Hydration enthalpy; Lattice energy

1 引言

自19世纪80年代以来, 室温离子液体(RTILs)作 为理想的反应溶剂、萃取溶剂、电解质材料等得
到科研工作者的高度关注. 而氨基酸因具有低成 本、生物降解性、无毒性等优点, Fukumoto等'在探 索新的RTILs过程中首次合成了以氨基酸为阴离子,

Received: July 30, 2015; Revised: September 10, 2015; Published on Web: September 11, 2015.

*Corresponding author. Email: guanweiy@sina.com.

The project was supported by the National Natural Science Foundation of China (21173107) and Liaoning Excellent Talents in University, China (2015025).

国家自然科学基金(21173107)和辽宁省高等学校优秀人才支持计划(2015025)资助项目

CEditorial office of Acta Physico-Chimica Sinica 
咪唑衍生物为阳离子的氨基酸离子液体(AAILs). AAILs 具有许多独特的性质: 即可用作手性溶剂, 能 够溶解多种生命物质, 吸附 $\mathrm{CO}_{2}$ 气体, 具有较强的氢 键网状结构等. ${ }^{2-6}$ 无论在生命科学、药物化学、环 境化学、还是化学工业等领域都具有广泛的应用 前景. ${ }^{7.8}$ AAILs已经得到了科研工作者的高度关注和 广泛研究. ${ }^{9-11}$

AAILs种类虽不断增加, 但其热化学数据, 如 标准摩尔溶解热、标准摩尔反应热、标准摩尔生 成热、标准摩尔燃烧热及水化热等还很少, 而这些 却是离子液体工业化过程中不论设备选型还是工 艺参数选定的重要基础物理化学数据. 为此, 本文 在前期研究 ${ }^{12-15}$ 的基础上, 做了如下工作: (1)通过中 和法合成了1-乙基-3-甲基咪唑丙氨酸离子液体, 采 用型号为 SRC 100的溶解-反应量热仪在 $(288.15 \pm$ $0.01)-(308.15 \pm 0.01) K$ 温度范围内每间隔5 K测定 了水中不同质量摩尔浓度的溶解焓 $\left(\Delta_{\mathrm{sol}} H_{\mathrm{m}}\right)$; (2)计算 各个温度下离子液体的标准摩尔溶解焓 $\left(\Delta_{\mathrm{sol}} H_{\mathrm{m}}^{\theta}\right)$, 不 同温度不同质量摩尔浓度的相对表观摩尔溶解焓 $\left({ }^{\oplus} L\right)$; (3)在 $298.15 \mathrm{~K}$ 下, 计算了 $\left[\mathrm{C}_{2} \mathrm{mim}\right][\mathrm{Ala}]$ 的格子 能 $U_{\mathrm{POT}}$ 和阴阳离子水化焓 $\left(\Delta H^{+}+\Delta H^{-}\right)$及阴离子水 化焓 $\Delta H^{-}\left([\mathrm{Ala}]^{-}\right)$, 同时也估算离子液体摩尔热容 $\left(C_{p}(\mathrm{IL})\right)$, 离子液体水溶液的的热容 $\left(C_{p(\mathrm{sol})}\right)$ 和表观摩 尔热容 $\left({ }^{\oplus} C_{p}\right)$.

\section{2 实验部分}

\section{1 实验材料}

离子交换树脂(717型, > 95\%), 用常规方法活化; 溴代乙烷, 丙氨酸(>98.5\%)用标准方法精制; $N$-甲 基咪唑(购于浙江双港化工厂), 使用前氮气保护减 压蒸馏; 氯化钾 (>99.99\%)、3-羟甲基胺基甲烷 (THAM，GR，> 99\%), 使用前真空干燥; 甲醇(> $99.8 \%)$ 、乙酸乙酯 $(>99.5 \%)$ 、乙腈 $(>99.5 \%)$ 、卡 尔・费休试剂, 均为AR试剂(购于国药集团化学试剂 有限公司); 高纯水由去离子水蒸馏得到; 离子液体
$\left[\mathrm{C}_{2} \operatorname{mim}\right][\mathrm{Ala}](>99 \%)$.

\section{2 离子液体 $\left[\mathrm{C}_{2} \mathrm{mim}\right][\mathrm{Ala}]$ 的合成}

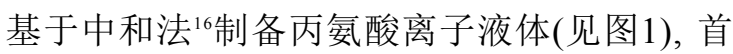
先根据文献 ${ }^{17}$ 合成了 1 - 乙基-3-甲基咪唑澳盐 ([ $\left.\left.\mathrm{C}_{2} \operatorname{mim}\right] \mathrm{Br}\right)$. 将 $N$-甲基咪唑粗产品在一定温度下提 纯, 再加入适量溴代正乙烷, 恒温 $\left(50-70{ }^{\circ} \mathrm{C}\right)$ 回流48 $\mathrm{h}$, 将产物冷藏 $12 \mathrm{~h}$ 后得到 $\left[\mathrm{C}_{2} \mathrm{mim}\right] \mathrm{Br}$ 白色晶体. 然后, 将其水溶液通过阴离子交换柱, 得到1-乙基-3-甲基 咪唑氢氧化物 $\left(\left[\mathrm{C}_{2} \mathrm{mim}\right] \mathrm{OH}\right)$ 溶液。最后, 将 $\left[\mathrm{C}_{2} \mathrm{mim}\right] \mathrm{OH}$ 与过量的丙氨酸水溶液反应 $48 \mathrm{~h}$, 依次 经减压蒸馏、过滤、干燥, 最终得到淡黄色离子液 体 $\left[\mathrm{C}_{2} \mathrm{mim}\right][\mathrm{Ala}]$. 经核磁共振氢谱( ${ }^{1} \mathrm{H}$ NMR)确定是 目标产物, 且差式扫描量热(DSC)分析表明该离子 液体无熔点(见Supporting Information中的图S1、 S2). 为减小样品中微量水的影响, 本文采用卡尔・费 休水分测定仪(ZSD-2 type)对目标产物中含水量进 行测定，其结果显示目标产物中含水量小于 $0.5 \%$ $(w)$, 硝酸银试剂检测产物无沉淀, 因此, 综合上述检 测结果可知产物纯度高于 $99 \%$.

\section{3 溶解焓的测定}

本文采用SRC 100型溶解反应量热仪 ${ }^{18-20}$ 测定离 子液体 $\left[\mathrm{C}_{2} \mathrm{mim}\right][\mathrm{Ala}]$ 溶解焓. 在使用仪器前选用氯 化钾和3-羟甲基胺基甲烷(THAM)在298.15 K下对 仪器进行校正, 将干燥过的氯化钾 (约 $0.3650 \mathrm{~g}$ ) 和 $\mathrm{THAM}($ 约 $0.5000 \mathrm{~g}$ ) 分别溶于高纯水和浓度为 0.1 $\mathrm{mol} \cdot \mathrm{dm}^{-3}$ 的稀盐酸溶液中, 并分别测定两种样品的 摩尔溶解焓. 测试结果表明氯化钾的摩尔溶解焓 $\Delta_{\mathrm{sol}} H_{\mathrm{m}}=(17.542 \pm 0.031) \mathrm{kJ} \cdot \mathrm{mol}^{-1}$, THAM的摩尔溶 解焓 $\Delta_{\mathrm{sol}} H_{\mathrm{m}}=(-29.794 \pm 0.028) \mathrm{kJ} \cdot \mathrm{mol}^{-1}$, 二者与文 献值 $\left(17.549 \text { 和 }-29.754 \mathrm{~kJ} \cdot \mathrm{mol}^{-1}\right)^{21,22}$ 较为接近, 实验 误差分别为 $0.04 \%$ 和 $0.14 \%$, 说明该溶解反应热量计 达到了实验要求. 然后, 用标定后的溶解反应热量 计分别测定了 $(288.15 \pm 0.01)-(308.15 \pm 0.01) \mathrm{K}$ 温 度范围内每间隔 $5 \mathrm{~K}$ 不同质量摩尔浓度离子液体 $\left[\mathrm{C}_{2} \mathrm{mim}\right][\mathrm{Ala}]$ 的摩尔溶解焓.

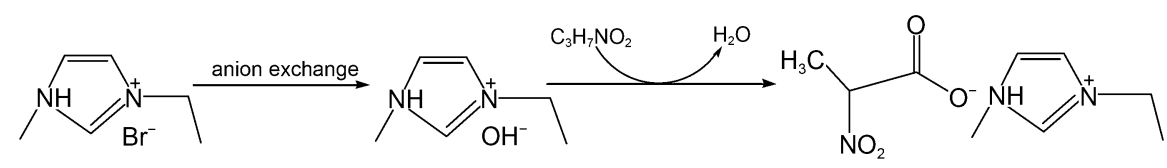

图1 中和方法制备 $\left[\mathrm{C}_{2} \mathrm{mim}\right][\mathrm{Ala}]$ 离子液体的过程

Fig.1 Synthesized process of ionic liquid (IL) $\left[\mathrm{C}_{2} \mathrm{mim}\right][\mathrm{Ala}]$ by the neutralization method 


\section{3 结果与讨论}

\section{1 离子液体的摩尔溶解焓}

本文在 $(288.15 \pm 0.01)-(308.15 \pm 0.01) \mathrm{K}$ 温度 范围内每间隔 $5 \mathrm{~K}$ 测定了不同质量摩尔浓度 $\left[\mathrm{C}_{2} \mathrm{mim}\right]$ [Ala] 的 $\Delta_{\mathrm{sol}} H_{\mathrm{m}}$, 其中标准不确定度 $u(T)= \pm 0.01 \mathrm{~K}$, $u(m)= \pm 0.0002 \mathrm{~g}, u(\Delta T)= \pm 0.0001 \mathrm{~K}$. 相关结果列 于表1-5.

从表中数据可以看出离子液体 $\left[\mathrm{C}_{2} \mathrm{mim}\right][\mathrm{Ala}]$ 溶 于水是一个典型的放热过程，且在同一温度下, 其 释放的热量随其质量摩尔浓度的增大而减小, 这与 我们曾报道的相关结果 ${ }^{15,23}$ 较好的一致.

\section{2 离子液体的标准摩尔溶解焓 $\left(\Delta_{\mathrm{sol}} \boldsymbol{H}_{\mathrm{m}}^{\boldsymbol{\theta}}\right)$}

参考Archer等 ${ }^{24}$ 的方法, 根据Debye-Hückel极限
方程, $\left[\mathrm{C}_{2} \mathrm{mim}\right][\mathrm{Ala}]$ 在不同温度下的标准摩尔溶解 焓可由下式得到:

$$
\begin{aligned}
Y & =\Delta_{\mathrm{sol}} H_{\mathrm{m}}-\left(A_{\mathrm{H}} / b\right) \ln \left(1+b I^{1 / 2}\right) \\
& =\Delta_{\mathrm{sol}} H_{\mathrm{m}}^{\theta}+\beta c
\end{aligned}
$$

式(1)中: $Y$ 为外推函数, 可由实验数据计算得到, $A_{\mathrm{H}}$ 为Debye-Hückel参数, ${ }^{25} b$ 为常数, $b=1.2,{ }^{25} c$ 为质 量摩尔浓度, $I$ 为离子液体强度(当电解质溶液按照 $1: 1$ 电解时, $I=c), \beta$ 为经验常数. 将外推函数 $Y$ 对质 量摩尔浓度 $c$ 作图, 可得一系列直线(见图2), 从直线 的截距和斜率可得到 $\Delta_{\mathrm{sol}} H_{\mathrm{m}}^{\theta}$ 和 $\beta$ 的值, 相关数据被列 于表6中. 通过表中数据可以看出标准摩尔溶解焓 随温度的升高而减小. 在 $298.15 \mathrm{~K}$ 下, [C $\left.\mathrm{C}_{2} \mathrm{mim}\right][\mathrm{Ala}]$

表1 $288.15 \mathrm{~K}$ 时离子液体 $\left[\mathrm{C}_{2} \mathrm{mim}\right][\mathrm{Ala}]$ 的摩尔溶解焓 $\left(\Delta_{\mathrm{sol}} H_{\mathrm{m}}\right)$, 外推函数 $(Y)$ 和相对表观摩尔溶解焓 $\left({ }^{\top} L\right)$ 值 Table 1 Values of the molar enthalpies of solution $\left(\Delta_{\mathrm{sol}} H_{\mathrm{m}}\right)$, the extrapolation function $(Y)$, and the apparent relative molar

\begin{tabular}{|c|c|c|c|c|c|}
\hline $\mathrm{m} / \mathrm{g}$ & $c /\left(\mathrm{mol} \cdot \mathrm{kg}^{-1}\right)$ & $-\Delta_{\mathrm{sol}} H_{\mathrm{m}} /\left(\mathrm{kJ} \cdot \mathrm{mol}^{-1}\right)$ & $\Delta T / \mathrm{K}$ & $-Y /\left(\mathrm{kJ} \cdot \mathrm{mol}^{-1}\right)$ & ${ }^{\Phi} L /\left(\mathrm{kJ} \cdot \mathrm{mol}^{-1}\right)$ \\
\hline 0.2364 & 0.01190 & $58.65 \pm 0.01$ & 0.0982 & $58.83 \pm 0.01$ & 1.2953 \\
\hline 0.2726 & 0.01372 & $58.51 \pm 0.02$ & 0.1263 & $58.69 \pm 0.02$ & 1.4396 \\
\hline 0.3066 & 0.01543 & $58.40 \pm 0.02$ & 0.1316 & $58.59 \pm 0.02$ & 1.5502 \\
\hline 0.3759 & 0.01891 & $58.17 \pm 0.02$ & 0.1608 & $58.38 \pm 0.02$ & 1.7787 \\
\hline 0.4050 & 0.02038 & $58.04 \pm 0.02$ & 0.1776 & $58.26 \pm 0.02$ & 1.9127 \\
\hline 0.4925 & 0.02478 & $57.69 \pm 0.03$ & 0.2064 & $57.93 \pm 0.03$ & 2.2604 \\
\hline 0.5357 & 0.02696 & $57.46 \pm 0.03$ & 0.2232 & $57.71 \pm 0.03$ & 2.4892 \\
\hline 0.5706 & 0.02871 & $57.23 \pm 0.03$ & 0.2437 & $57.48 \pm 0.03$ & 2.7247 \\
\hline 0.6081 & 0.03060 & $57.01 \pm 0.03$ & 0.2600 & $57.28 \pm 0.03$ & 2.9363 \\
\hline 0.6753 & 0.03398 & $56.73 \pm 0.04$ & 0.2854 & $57.01 \pm 0.04$ & 3.2205 \\
\hline 0.7424 & 0.03736 & $56.41 \pm 0.04$ & 0.3144 & $56.70 \pm 0.04$ & 3.5376 \\
\hline 0.8012 & 0.04031 & $56.10 \pm 0.04$ & 0.3390 & $56.40 \pm 0.04$ & 3.8477 \\
\hline
\end{tabular}
enthalpy $\left({ }^{\Phi} L\right)$ for $\left[C_{2}\right.$ mim] [Ala] at $288.15 \mathrm{~K}$

$m$ : sample mass, $c$ : molality, $\Delta T$ : temperature difference; measured at atmospheric pressure

表2 293.15 K时离子液体 $\left[\mathrm{C}_{2} \mathrm{mim}\right][\mathrm{Ala}]$ 的 $\Delta_{\mathrm{sol}} H_{\mathrm{m}}, Y$ 和 ${ }^{\Phi} L$ 值

\begin{tabular}{|c|c|c|c|c|c|}
\hline$m / g$ & $c /\left(\mathrm{mol} \cdot \mathrm{kg}^{-1}\right)$ & $-\Delta_{\mathrm{sol}} H_{\mathrm{m}} /\left(\mathrm{kJ} \cdot \mathrm{mol}^{-1}\right)$ & $\Delta T / \mathrm{K}$ & $-Y /\left(\mathrm{kJ} \cdot \mathrm{mol}^{-1}\right)$ & ${ }^{\Phi} L /\left(\mathrm{kJ} \cdot \mathrm{mol}^{-1}\right)$ \\
\hline 0.2080 & 0.01047 & $55.56 \pm 0.01$ & 0.0742 & $55.74 \pm 0.01$ & 1.1103 \\
\hline 0.2393 & 0.01204 & $55.41 \pm 0.01$ & 0.0863 & $55.60 \pm 0.01$ & 1.2556 \\
\hline 0.2563 & 0.01290 & $55.38 \pm 0.01$ & 0.1076 & $55.57 \pm 0.01$ & 1.2902 \\
\hline 0.3223 & 0.01622 & $55.03 \pm 0.02$ & 0.1352 & $55.25 \pm 0.02$ & 1.6387 \\
\hline 0.3772 & 0.01898 & $54.82 \pm 0.02$ & 0.1480 & $55.05 \pm 0.02$ & 1.8525 \\
\hline 0.4216 & 0.02121 & $54.57 \pm 0.02$ & 0.1904 & $54.82 \pm 0.02$ & 2.0983 \\
\hline 0.4726 & 0.02378 & $54.35 \pm 0.03$ & 0.2072 & $54.61 \pm 0.03$ & 2.3206 \\
\hline 0.5043 & 0.02538 & $54.21 \pm 0.03$ & 0.2117 & $54.48 \pm 0.03$ & 2.4605 \\
\hline 0.5759 & 0.02898 & $53.90 \pm 0.03$ & 0.2296 & $54.18 \pm 0.03$ & 2.7741 \\
\hline 0.6274 & 0.03157 & $53.63 \pm 0.03$ & 0.2526 & $53.93 \pm 0.03$ & 3.0359 \\
\hline 0.6959 & 0.03502 & $53.31 \pm 0.04$ & 0.2872 & $53.62 \pm 0.04$ & 3.3583 \\
\hline 0.7423 & 0.03735 & $53.09 \pm 0.04$ & 0.3053 & $53.41 \pm 0.04$ & 3.5821 \\
\hline
\end{tabular}

Table 2 Values of $\Delta_{\mathrm{sol}} H_{\mathrm{m}}, Y$, and ${ }^{\Phi} L$ for $\left[\mathrm{C}_{2} \mathrm{mim}\right][\mathrm{Ala}]$ at $293.15 \mathrm{~K}$ 
表3 298.15 K时离子液体 $\left[C_{2} \mathrm{mim}\right]\left[\right.$ [Ala]的 $\Delta_{\text {sol }} H_{\mathrm{m}}, Y$ 和 ${ }^{\Phi} L$ 值

Table 3 Values of the $\Delta_{\text {sol }} H_{\mathrm{m}}, Y$, and ${ }^{\Phi} L$ for $\left[\mathrm{C}_{2} \mathrm{mim}\right.$ ][Ala] at $298.15 \mathrm{~K}$

\begin{tabular}{ccccc}
\hline$m / \mathrm{g}$ & $c /\left(\mathrm{mol} \cdot \mathrm{kg}^{-1}\right)$ & $-\Delta_{\text {sol }} H_{\mathrm{m}} /\left(\mathrm{kJ} \cdot \mathrm{mol}^{-1}\right)$ & $\Delta T / \mathrm{K}$ & $-Y /\left(\mathrm{kJ} \cdot \mathrm{mol}{ }^{-1}\right)$ \\
\hline 0.2267 & 0.01141 & $53.46 \pm 0.01$ & 0.0684 & $53.64 \pm 0.01$ \\
0.2567 & 0.01292 & $53.31 \pm 0.01$ & 0.0905 & $53.50 \pm 0.01$ \\
0.3034 & 0.01527 & $53.07 \pm 0.01$ & 0.1146 & $53.28 \pm 0.01$ \\
0.3460 & 0.01741 & $52.92 \pm 0.02$ & 0.1344 & $53.14 \pm 0.02$ \\
0.4368 & 0.02198 & $52.60 \pm 0.02$ & 0.1523 & $52.85 \pm 0.02$ \\
0.4809 & 0.02420 & $52.43 \pm 0.02$ & 0.1820 & $52.69 \pm 0.02$ \\
0.5463 & 0.02749 & $52.20 \pm 0.03$ & 0.2046 & $52.48 \pm 0.03$ \\
0.5979 & 0.03008 & $52.03 \pm 0.03$ & 0.2314 & $52.32 \pm 0.03$ \\
0.6402 & 0.03221 & $51.86 \pm 0.03$ & 0.2488 & $52.105 \pm 0.03$ \\
0.6875 & 0.03459 & $51.68 \pm 0.03$ & 0.2718 & 2.2182 \\
0.7412 & 0.03730 & $51.44 \pm 0.04$ & 0.2886 & $51.98 \pm 0.03$ \\
0.7627 & 0.03838 & $51.27 \pm 0.04$ & 0.3059 & $51.76 \pm 0.04$ \\
\hline
\end{tabular}

measured at atmospheric pressure

表4 303.15 K时离子液体 $\left[C_{2} \mathrm{mim}\right][\mathrm{Ala}]$ 的 $\Delta_{\mathrm{sol}} H_{\mathrm{m}}, Y$ 和 ${ }^{\Phi} L$ 值

Table 4 Values of the $\Delta_{\text {sol }} H_{\mathrm{m}}, Y$, and ${ }^{\Phi} L$ for $\left[C_{2}\right.$ mim] [Ala] at $298.15 \mathrm{~K}$

\begin{tabular}{|c|c|c|c|c|c|}
\hline $\mathrm{m} / \mathrm{g}$ & $c /\left(\mathrm{mol} \cdot \mathrm{kg}^{-1}\right)$ & $-\Delta_{\mathrm{sol}} H_{\mathrm{m}} /\left(\mathrm{kJ} \cdot \mathrm{mol}^{-1}\right)$ & $\Delta T / \mathrm{K}$ & $-Y /\left(\mathrm{kJ} \cdot \mathrm{mol}^{-1}\right)$ & ${ }^{\Phi} L /\left(\mathrm{kJ} \cdot \mathrm{mol}^{-1}\right)$ \\
\hline 0.2263 & 0.01139 & $50.68 \pm 0.01$ & 0.0542 & $50.88 \pm 0.01$ & 0.7941 \\
\hline 0.2645 & 0.01331 & $50.49 \pm 0.01$ & 0.0653 & $50.70 \pm 0.01$ & 0.9802 \\
\hline 0.3184 & 0.01602 & $50.30 \pm 0.01$ & 0.0978 & $50.54 \pm 0.01$ & 1.1687 \\
\hline 0.3828 & 0.01926 & $50.08 \pm 0.02$ & 0.1172 & $50.33 \pm 0.02$ & 1.3904 \\
\hline 0.4346 & 0.02187 & $49.93 \pm 0.02$ & 0.1314 & $50.20 \pm 0.02$ & 1.5397 \\
\hline 0.4719 & 0.02374 & $49.72 \pm 0.02$ & 0.1453 & $50.00 \pm 0.02$ & 1.7526 \\
\hline 0.5521 & 0.02778 & $49.46 \pm 0.02$ & 0.1786 & $49.77 \pm 0.02$ & 2.0063 \\
\hline 0.5716 & 0.02876 & $49.50 \pm 0.02$ & 0.1868 & $49.81 \pm 0.02$ & 1.9681 \\
\hline 0.6203 & 0.03121 & $49.35 \pm 0.03$ & 0.2023 & $49.67 \pm 0.03$ & 2.1185 \\
\hline 0.6724 & 0.03383 & $49.19 \pm 0.03$ & 0.2184 & $49.52 \pm 0.03$ & 2.2756 \\
\hline 0.7591 & 0.03820 & $48.95 \pm 0.03$ & 0.2494 & $49.30 \pm 0.03$ & 2.5213 \\
\hline 0.7927 & 0.03989 & $48.80 \pm 0.04$ & 0.2787 & $49.15 \pm 0.04$ & 2.6729 \\
\hline
\end{tabular}

表5 308.15 K时离子液体 $\left[C_{2} \operatorname{mim}\right][\mathrm{Ala}]$ 的 $\Delta_{\mathrm{sol}} H_{\mathrm{m}}, Y$ 和 ${ }^{\Phi} L$ 值

Table 5 Values of the $\Delta_{\text {sol }} H_{\mathrm{m}}, Y$, and ${ }^{\Phi} L$ for $\left[\mathrm{C}_{2} \mathrm{mim}\right.$ ][Ala] at $298.15 \mathrm{~K}$

\begin{tabular}{ccccc}
\hline$m / \mathrm{g}$ & $c /\left(\mathrm{mol} \cdot \mathrm{kg}^{-1}\right)$ & $-\Delta_{\mathrm{sol}} H_{\mathrm{m}} /\left(\mathrm{kJ} \cdot \mathrm{mol}^{-1}\right)$ & $\Delta T / \mathrm{K}$ & $-Y /\left(\mathrm{kJ} \cdot \mathrm{mol}^{-1}\right)$ \\
\hline 0.2659 & 0.01338 & $49.06 \pm 0.01$ & 0.0516 & $49.27 \pm 0.01$ \\
0.3173 & 0.01597 & $48.91 \pm 0.01$ & 0.0794 & $49.15 \pm 0.01$ \\
0.3479 & 0.01751 & $48.80 \pm 0.01$ & 0.0845 & $49.04 \pm 0.01$ \\
0.4361 & 0.02194 & $48.46 \pm 0.02$ & 0.1174 & $48.73 \pm 0.02$ \\
0.4750 & 0.02390 & $48.28 \pm 0.02$ & 0.1307 & $48.56 \pm 0.02$ \\
0.5180 & 0.02606 & $48.19 \pm 0.02$ & 0.1498 & $48.49 \pm 0.02$ \\
0.5947 & 0.02992 & $47.91 \pm 0.02$ & 0.1907 & $48.22 \pm 0.02$ \\
0.6253 & 0.03146 & $47.74 \pm 0.03$ & 0.1971 & $48.06 \pm 0.03$ \\
0.6959 & 0.03502 & $47.46 \pm 0.03$ & 0.2180 & 4.9844 \\
0.7424 & 0.03736 & $47.28 \pm 0.03$ & 0.2378 & 2.0673 \\
0.7977 & 0.04014 & $47.10 \pm 0.03$ & 0.2623 & $47.79 \pm 0.03$ \\
0.8424 & 0.04239 & $46.89 \pm 0.03$ & 0.2731 & 2.5183 \\
\hline
\end{tabular}




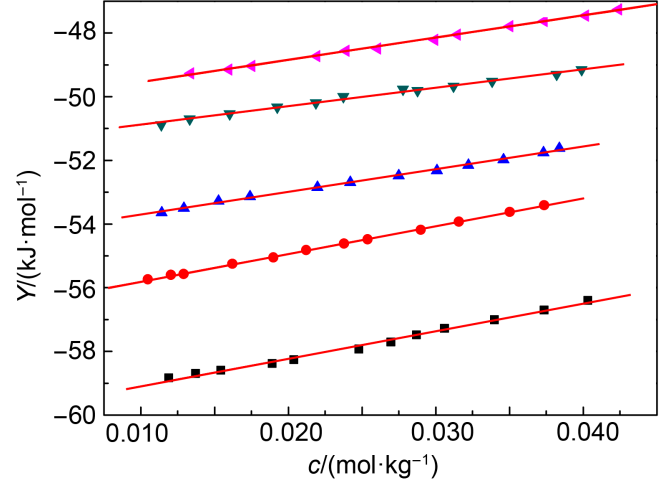

图2 288.15-308.15 K温区内离子液体 $\left[\mathrm{C}_{2} \mathrm{mim}\right]$ [Ala]的 $Y$ 对 $c$ 的拟合图

Fig.2 Plots of the extrapolation function $Y$ against $c$ for IL [ $\left.\mathrm{C}_{2} \mathrm{mim}\right][\mathrm{Ala}]$ in temperature range of 288.15 to $308.15 \mathrm{~K}$

-288.15 K; • •293.15 K; $\Delta 298.15 \mathrm{~K} ; \quad \nabla 303.15 \mathrm{~K} ; \quad 4308.15 \mathrm{~K}$

的标准摩尔溶解焓值: $\Delta_{\mathrm{sol}} H_{\mathrm{m}}^{\theta}=-54.42 \mathrm{~kJ} \cdot \mathrm{mol}^{-1}$, 小 于 $\left[\mathrm{C}_{4} \mathrm{mim}\right][\mathrm{Ala}]$ 的值: $\Delta_{\mathrm{sol}} H_{\mathrm{m}}^{\theta}=-61.42 \mathrm{~kJ} \cdot \mathrm{mol}^{-1}{ }^{26}$ 随 着咪唑烷基碳链 $\left(-\mathrm{CH}_{2}-\right)$ 的增加标准摩尔溶解焓 增大, 这与我们实验过程中观察到的 $\left[\mathrm{C}_{2} \mathrm{mim}\right][\mathrm{Ala}]$

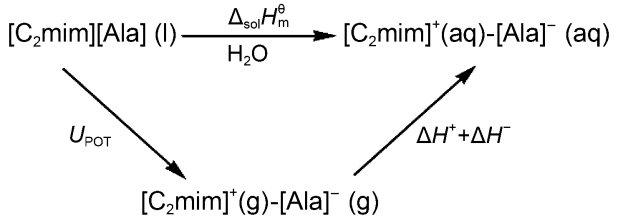

图3 离子液体 $\left[\mathrm{C}_{2} \mathrm{mim}\right][\mathrm{Ala}]$ 水化焓的热力学循环图

Fig.3 Thermodynamic cycle for estimation the values of the hydration enthalpy of IL [ $\left.\mathrm{C}_{2} \mathrm{mim}\right][\mathrm{Ala}]$

在溶解过程中放热较小是一致的.

离子液体 $\left[\mathrm{C}_{2} \mathrm{mim}\right][\mathrm{Ala}]$ 的相对表观摩尔焓 $\left({ }^{\oplus} L\right)$ 可由方程(2)计算得到, 计算的结果见表1-5.

$$
{ }^{\Phi} L=\Delta_{\text {sol }} H_{\mathrm{m}}-\Delta_{\text {sol }} H_{\mathrm{m}}^{\theta}
$$

\section{3 离子液体的阴阳离子水化焓的估算}

通过设计热力学循环, 如图3所示, 离子液体阴 阳离子水化焓值可由下式计算得到:

$$
\left(\Delta H^{+}+\Delta H^{-}\right)=\Delta_{\mathrm{sol}} H_{\mathrm{m}}^{\theta}-U_{\text {РОт }}
$$

式中: $\Delta H^{-}$和 $\Delta H^{+}$分别为阴、阳离子水化焓, $U_{\mathrm{POT}}$ 为 离子液体的格子能.

表6 288.15-308.15 K温度范围离子液体 $\left[\mathrm{C}_{2} \mathrm{mim}\right][\mathrm{Ala}]$ 的标准摩尔溶解焓 $\left(\Delta_{\mathrm{sol}} H_{\mathrm{m}}^{\mathbf{0}}\right)$ 值

Table 6 Values of standard molar solution enthalpy $\left(\Delta_{\text {sol }} H_{m}^{0}\right)$ for $\left[\mathrm{C}_{2} \mathrm{mim}\right][\mathrm{Ala}]$ in temperature range of 288.15-308.15 K

\begin{tabular}{cccc}
\hline$T / \mathrm{K}$ & $-\Delta_{\text {sol }} H_{\mathrm{m}}{ }^{\theta} /\left(\mathrm{kJ} \cdot \mathrm{mol}^{-1}\right)$ & $\beta /\left(\mathrm{kJ} \cdot \mathrm{kg} \cdot \mathrm{mol}^{-2}\right)$ & $s /\left(\mathrm{kJ} \cdot \mathrm{mol}^{-1}\right)$ \\
288.15 & $59.95 \pm 0.06$ & 86.10 & 0.07 \\
293.15 & $56.67 \pm 0.02$ & 86.77 & 0.02 \\
298.15 & $54.42 \pm 0.03$ & 71.14 & 0.996 \\
303.15 & $51.47 \pm 0.04$ & 58.18 & 0.999 \\
308.15 & $50.26 \pm 0.03$ & 70.01 & 0.099 \\
\hline
\end{tabular}

$\beta$ is empirical constant, $s$ is standard deviation, $r$ is correlation coefficient.

表7 离子液体 $\left[\mathrm{C}_{2} \mathrm{mim}\right][\mathrm{Ala}]$ 不同质量摩尔浓度下表观摩尔热容 $\left({ }^{\Phi} C_{p}\right)$ 和溶液的热容 $\left(C_{p(\mathrm{sol})}\right)$ 值

Table 7 Values of the apparent molar heat capacity $\left({ }^{\Phi} C_{p}\right)$ and the heat capacity of aqueous $\left(C_{p(\text { sol })}\right)$ with different

\begin{tabular}{|c|c|c|c|c|c|c|c|c|c|}
\hline \multirow{2}{*}{$c /\left(\mathrm{mol} \cdot \mathrm{kg}^{-1}\right)$} & \multicolumn{5}{|c|}{$-\Delta_{\mathrm{sol}} H_{\mathrm{m}} /\left(\mathrm{kJ} \cdot \mathrm{mol}^{-1}\right)$} & \multirow{2}{*}{$\left(\partial \Delta_{\mathrm{sol}} H_{\mathrm{m}} / \partial T\right)_{p, \mathrm{~m}} /\left(\mathrm{J} \cdot \mathrm{K}^{-1} \cdot \mathrm{mol}^{-1}\right)$} & \multirow{2}{*}{$r$} & \multirow{2}{*}{${ }^{\Phi} C_{p} /\left(\mathrm{J} \cdot \mathrm{K}^{-1} \cdot \mathrm{mol}^{-1}\right)$} & \multirow{2}{*}{$C_{p(\mathrm{sol})} /\left(\mathrm{J} \cdot \mathrm{kg}^{-1} \cdot \mathrm{K}^{-1}\right.$} \\
\hline & $288.15 \mathrm{~K}$ & $293.15 \mathrm{~K}$ & $298.15 \mathrm{~K}$ & $303.15 \mathrm{~K}$ & $308.15 \mathrm{~K}$ & & & & \\
\hline 0.01500 & 58.49 & 55.20 & 53.18 & 50.43 & 49.04 & 473.37 & 0.9919 & 817.9 & 432.3 \\
\hline 0.02100 & 57.96 & 54.67 & 52.74 & 50.07 & 48.61 & 466.08 & 0.9923 & 810.6 & 437.0 \\
\hline 0.02300 & 57.78 & 54.48 & 52.59 & 49.94 & 48.46 & 463.65 & 0.9924 & 808.1 & 438.6 \\
\hline 0.02600 & 57.50 & 54.20 & 52.36 & 49.75 & 48.23 & 460.00 & 0.9925 & 804.5 & 440.9 \\
\hline 0.02800 & 57.32 & 54.02 & 52.21 & 49.62 & 48.08 & 457.57 & 0.9925 & 802.1 & 442.5 \\
\hline 0.03100 & 57.04 & 53.74 & 51.98 & 49.43 & 47.85 & 453.92 & 0.9926 & 798.4 & 444.8 \\
\hline 0.03400 & 56.77 & 53.47 & 51.75 & 49.24 & 47.63 & 450.28 & 0.9926 & 794.8 & 447.0 \\
\hline 0.03500 & 56.68 & 53.38 & 51.67 & 49.18 & 47.55 & 449.06 & 0.9926 & 793.6 & 447.8 \\
\hline 0.03700 & 56.50 & 53.20 & 51.52 & 49.05 & 47.41 & 446.63 & 0.9925 & 791.1 & 449.3 \\
\hline 0.04000 & 56.23 & 52.92 & 51.30 & 48.87 & 47.18 & 442.98 & 0.9925 & 787.5 & 451.5 \\
\hline 0.04400 & 55.87 & 52.56 & 51.00 & 48.62 & 46.89 & 438.12 & 0.9923 & 782.6 & 454.4 \\
\hline 0.04600 & 55.69 & 52.38 & 50.85 & 48.49 & 46.74 & 435.69 & 0.9922 & 780.2 & 455.9 \\
\hline
\end{tabular}
molalities for IL [ $\mathrm{C}_{2}$ mim][Ala] 
利用Glasser ${ }^{27,28}$ 经验方程可计算离子液体的格 子能:

$$
U_{\mathrm{POT}}=2\left(\alpha V_{\mathrm{m}}^{-1 / 3}+\beta\right)
$$

式中: $\alpha$ 和 $\beta$ 是常数, ${ }^{29} \alpha=83.3 \mathrm{~nm} \cdot \mathrm{kJ} \cdot \mathrm{mol}^{-1}, \beta=157.3$ $\mathrm{kJ} \cdot \mathrm{mol}^{-1}, V_{\mathrm{m}}$ 为 $\left[\mathrm{C}_{2} \mathrm{mim}\right][\mathrm{Ala}]$ 的分子体积. 在 298.15 $\mathrm{K}$ 下, $V_{\mathrm{m}}=0.2914 \mathrm{~nm}^{3},{ }^{30}$ 得到 $U_{\text {POT }}=566 \mathrm{~kJ} \cdot \mathrm{mol}^{-1}$.

根据方程(3), 计算[ $\left.\mathrm{C}_{2} \mathrm{mim}\right][\mathrm{Ala}]$ 的阴、阳离子水化 焓 $\left(\Delta H^{+}+\Delta H^{-}\right)=-620 \mathrm{~kJ} \cdot \mathrm{mol}^{-1}$. 由于 $\left(\Delta H^{+}+\Delta H^{-}\right)=$ $\Delta H^{+}+\Delta H^{-}$, 其中阳离子水化焓 $\Delta H^{+}\left(\left[\mathrm{C}_{2} \mathrm{mim}\right]^{+}\right)=$ $-233 \mathrm{~kJ} \cdot \mathrm{mol}^{-1}, 31$ 因此, $\Delta H^{-}\left([\mathrm{Ala}]^{-}\right)=-387 \mathrm{~kJ} \cdot \mathrm{mol}^{-1}$.

\section{4 离子液体表观摩尔热容 $\left({ }^{\top} C_{p}\right)$}

离子液体 $\left[\mathrm{C}_{2} \mathrm{mim}\right][\mathrm{Ala}]$ 的表观摩尔热容 ${ }^{\oplus} C_{p}$ 由方 程(5)计算得到:

$$
{ }^{\Phi} C_{p}=\left(\partial \Delta_{\mathrm{sol}} H_{\mathrm{m}} / \partial T\right)_{p, \mathrm{~m}}+C_{p}(\mathrm{ILs})
$$

根据Archer等 ${ }^{24}$ 的方法, 计算了 $\left[\mathrm{C}_{2} \mathrm{mim}\right][\mathrm{Ala}]$ 在 一定质量摩尔浓度下的摩尔溶解焓 $\Delta_{\mathrm{sol}} H_{\mathrm{m}}$, 并将 $\Delta_{\mathrm{sol}} H_{\mathrm{m}}$ 对 $T$ 作线性拟合图, 所得直线的斜率即为 $\left(\partial \Delta_{\mathrm{sol}} H_{\mathrm{m}} / \partial T\right)_{p, \mathrm{~m}}$, 这些结果均被列于表7中. 此外, 离子 液体的摩尔热容 $C_{p}(\mathrm{ILs})$ 可根据Paulechka等 ${ }^{2}$ 提出得 经验方程进行估算:

$$
\begin{aligned}
& C_{p}(\mathrm{ILs})=8.6+1.915 \mathrm{~V} \\
& { }^{\Phi} C_{p}=\left(C_{p}(\mathrm{sol})-1000 C_{p, \mathrm{~m}}^{0} / M_{\mathrm{w}}\right) / c
\end{aligned}
$$

其中: $V$ 为 $\left[\mathrm{C}_{2} \mathrm{mim}\right][\mathrm{Ala}]$ 的摩尔体积, $V=175.4 \mathrm{~cm}^{3}$, $C_{p, \mathrm{~m}}^{0}$ 为纯水的摩尔热容, $M_{\mathrm{w}}$ 为水的摩尔质量. 根据 方程(6), 离子液体 $\left[\mathrm{C}_{2} \mathrm{mim}\right][\mathrm{Ala}]$ 的摩尔热容 $C_{p}(\mathrm{ILs})=$ $344.49 \mathrm{~J} \cdot \mathrm{K}^{-1} \cdot \mathrm{mol}^{-1}$, 将结果带入方程(5), 得到了不同 质量摩尔浓度下的 ${ }^{\Phi} C_{p}$. 将上述 ${ }^{\oplus} C_{p}$ 及其相对应的 $c$ 值 代入方程(7)可计算出溶液的热容 $C_{p(\mathrm{sol})}$, 上述计算的 结果均被列于表7中.

\section{4 结 论}

在 $288.15 \mathrm{~K}$ 至 $308.15 \mathrm{~K}$ 温度范围内每隔 $5 \mathrm{~K}$, 测 定了离子液体的摩尔溶解焓 $\Delta_{\mathrm{sol}} H_{\mathrm{m}}$, 并计算了标准摩 尔溶解焓 $\Delta_{\mathrm{sol}} H_{\mathrm{m}}^{\theta}$ 和相对表观摩尔溶解焓 ${ }^{\circledR} L$, 且通过 数据得出标准摩尔溶解焓值随温度的升高而减小, 通过对 $\left[\mathrm{C}_{2} \mathrm{mim}\right][\mathrm{Ala}]$ 和 $\left[\mathrm{C}_{4} \mathrm{mim}\right][\mathrm{Ala}]$ 标准摩尔溶解 焓值的对比发现随着咪唑烷基碳链 $\left(-\mathrm{CH}_{2}-\right)$ 的增 加标准摩尔溶解焓逐渐增大; 计算了 $298.15 \mathrm{~K}$ 下 $\left[\mathrm{C}_{2} \mathrm{mim}\right][\mathrm{Ala}]$ 的阴阳离子水化焓 $\left(\Delta H^{+}+\Delta H^{-}\right)$和阴离 子水化焓 $\Delta H^{-}\left([\mathrm{Ala}]^{-}\right)$; 此外, 估算了离子液体水溶液 的热容和不同质量摩尔浓度的表观摩尔热容.
Supporting Information: available free of charge via the internet at http://www.whxb.pku.edn.cn

\section{References}

(1) Fukumoto, K.; Yoshizawa, M.; Ohno, H. J. Am. Chem. Soc. 2005, 127, 2398. doi: 10.1021/ja043451i

(2) Ohira, K.; Yoshida, K.; Hayase, S.; Itoh, T. Chemistry Letters 2012, 41, 987. doi: 10.1246/c1.2012.987

(3) Rauta, D. G.; Sundman, O.; Su, W.; Virtanen, P.; Sugano, Y.; Kordasd, K.; Mikkola, J. P. Carbohydrate Polymer 2015, 130 , 18. doi: 10.1016/j.carbpol.2015.04.032

(4) Pemberton, W. J.; Droessler, J. E.; Kinyanjui, J. M.; Czerwinski, K. R.; Hatchett, D. W. Electrochimica Acta 2013, 93, 264. doi: 10.1016/j.electacta.2013.01.044

(5) Pinto, A. M.; Rodríguez, H.; Arce, A.; Soto, A. Journal of Chemical Thermodynamics 2014, 77, 197. doi: 10.1016/j.jct.2013.10.023

(6) Christodoulou, C. P. K.; Stavrou, I. J.; Mavroudi, M. C. Journal of Chromatography A 2014, 1363, 2. doi: 10.1016/j.chroma.2014.05.059

(7) Kasahara, S.; Kamio, E.; Matsuyama, H. J. Membrane Science 2014, 454, 155. doi: 10.1016/j.memsci.2013.12.009

(8) Li, X. H.; Jiang, Y. B.; Zhang, L.; Li, R. Acta Phys. -Chim. Sin. 2006, 22, 747. [李雪辉, 江燕斌, 张 否, 李 榕. 物理化学学 报, 2006, 22, 747.] doi: 10.3866/PKU.WHXB20060620

(9) Primerano, P.; Milazzo, M. F.; Risitano, F.; Matarazzo, A. Journal of Chemical Technology and Biotechnology 2015, doi: $10.1002 /$ jctb. 4717

(10) Liu, Y.; Tian, A.; Wang, X.; Qi, J.; Wang, F.; Ma, Y.; Ito, Y.; Wei, Y. Journal of Chromatography A 2015, 1400, 40. doi: 10.1016/j.chroma.2015.04.045

(11) Bi, Y. H.; Duan, Z. Q.; Li, X. Q.; Wang, Z. Y.; Zhao, X. R. Journal of Agricultural and Food Chemistry 2015, 63, 558. doi: 10.1021/jf505296k

(12) Wei, J.; Ma, T. Y.; Ma, X. X.; Guan, W.; Liu, Q. S.; Yang, J. Z. RSC Adv. 2014, 4, 30725. doi: 10.1039/C4RA04391J

(13) Guan, W.; Yang, J. Z.; Li, L.; Wang, H.; Zhang, Q. G. Fluid Phase Equilibria 2006, 239, 161. doi: 10.1016/j.fluid. 2005.11.015

(14) Ma, X. X.; Wei, J.; Zhang, Q. B.; Tian, F.; Feng, Y. Y.; Guan, W. Ind. Eng. Chem. Res. 2013, 52, 9490. doi: 10.1021/ie401130d

(15) Wei, J.; Zhang, Q. B.; Tian, F.; Zheng, L.; Guan, W.; Yang, J. Z. Fluid Phase Equilibria 2014, 371, 1. doi: 10.1016/ j.fluid.2014.03.011

(16) Fukumoto, K.; Yoshizawa, M.; Ohno, H. J. Am. Chem. Soc. 2005, 127, 2398. doi: 10.1021/ja043451i

(17) Wilkes, J. S.; Levisky, J. A.; Wilson, R. A.; Hussey, C. L. Inorg. Chem. 1982, 21, 1263. doi: 10.1021/ic00133a078

(18) Di, Y. Y.; Qu, S. S.; Liu, Y.; Wen, D. C.; Tang, H.; Li, L. W. Thermochim Acta 2002, 387, 115. doi: 10.1016/S0040-6031 (01)00831-0 
(19) Liu, J. G.; Xue, W. F.; Qin, Y.; Yan, C. W. J. Chem. Eng. Data 2009, 54, 1938. doi: 10.1021/je8009557

(20) Ji, M.; Liu, M. Y.; Gao, S. L.; Shi, Q. Z. Instrum. Sci. Technol. 2001, 29, 53. doi: 10.1081/CI-100001408

(21) Rychly, R.; Pekarek, V. J. Chem. Thermodyn. 1977, 9, 391. doi: 10.1016/0021-9614(77)90060-X

(22) Montgomery, R. L.; Melaugh, R. A.; Lau, C. C.; Meier, G. H.; Chan, H. H.; Rossini, F. D. J. Chem. Thermodyn. 1977, 9 , 915. doi: 10.1016/0021-9614(77)90214-2

(23) Ma, X. X.; Li, L.; Wei, J.; Duan, W. B.; Guan, W.; Yang, J. Z J. Chem. Eng. Data 2012, 57, 3171. doi: 10.1021/je300768j

(24) Archer, D. G.; Widegren, J. A.; Kirklin, D. R.; Magee, J. W J. Chem. Eng. Data 2005, 50, 1484. doi: 10.1021/je050136i

(25) Pitzer, K. S. Activity Coefficients in Electrolyte Solutions, Chapter 3; CRC Press: Boca Raton, FL. 1991.

(26) Fang, D. W.; Guan, W.; Tong, J.; Wang, Z. W.; Yang, J. Z. J. Phys. Chem. B 2008, 112, 7499. doi: 10.1021/jp801269u
(27) Glasser, L. Thermochim. Acta 2004, 421, 87. doi: 10.1016/j.tca.2004.03.015

(28) Glasser, L. Jenkins, H. D. B. J. Chem. Eng. Data 2011, 56, 874. doi: 10.1021/je100683u

(29) Gutowski, K. E.; Rogers, R. D.; Dixon, D. A. J. Phys. Chem. B 2007, 111, 4788. doi: 10.1021/jp066420d

(30) Zhang, Q. B. Synthesis and Study on the Physico-Chemical Properties of the Acetic Acid Series and Alanine Series of Ionic Liquids. Master Dissertation, Liaoning University, Shenyang, 2014. [张秋波. 醋酸离子液体和丙氨酸离子液体的合成及物 理化学性质的测定[D]. 沈阳: 辽宁大学, 2014.]

(31) Yang, J. Z.; Zhang, Z. H.; Fang, D. W.; Li, J. G.; Guan, W. Fluid Phase Equilibria 2006, 247, 80. doi: 10.1016/j.fluid.2006.06.016

(32) Paulechka, Y. U.; Kabo, A. G.; Blokhin, A. V.; Kabo, G. J.; Shevelyova, M. P. J. Chem. Eng. Data 2010, 55, 2719. doi: 10.1021/je900974u 
Supplementary Information for Acta Phys. -Chim. Sin. 2015, 31 (11), 2036-2042 doi: 10.3866/PKU.WHXB201509111

\title{
1-乙基-3-甲基咪唑丙氨酸离子液体热力学性质的研究
}

\author{
郑 玲 $^{1}$ 柇本汉 ${ }^{1} \quad$ 卜晓雪 $^{1}$ 潘 $^{\text {懿 }^{1}}$ 董家新 $^{2}$ \\ 关 伟 1 ,* \\ ( ${ }^{1}$ 辽宁大学化学院, 沈阳 110036; 2 广西师范大学化学化工学院, 广西 桂林 541004)
}

\section{Study on Thermodynamic Properties of Ionic Liquid 1-Ethyl-3-methylimidazolium Alanine}

\author{
ZHENG Ling $^{1} \quad$ FAN Ben-Han $^{1} \quad$ BU Xiao-Xue $^{1} \quad$ PAN Yi $^{1} \quad$ DONG Jia-Xin ${ }^{2}$ \\ GUAN Wei ${ }^{1, *}$ \\ ( ${ }^{1}$ College of Chemistry, Liaoning University, Shenyang 110036, P. R. China; ${ }^{2}$ College of Chemistry \& Chemical \\ Engineering, Guangxi Normal University, Guilin 541004, Guangxi Province, P. R. China) \\ *Corresponding author. Email: guanweiy@sina.com.
}




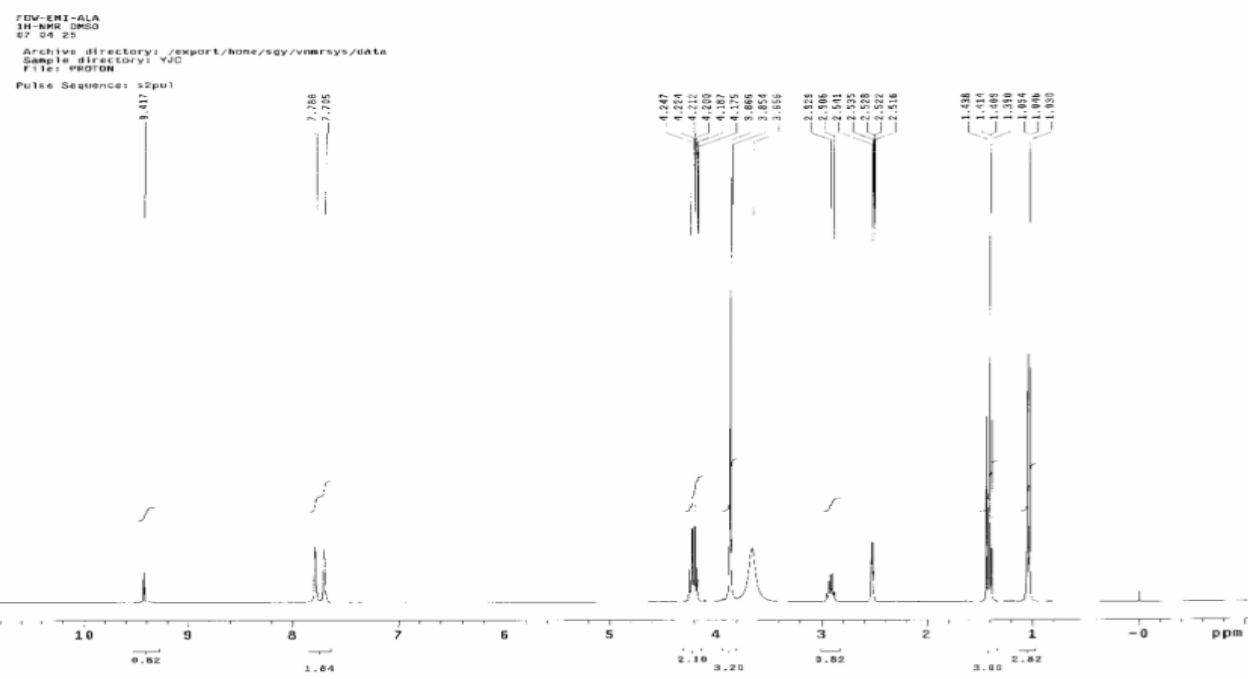

Fig.S1 离子液体 $\left[\mathrm{C}_{2} \mathrm{mim}\right][\mathrm{Ala}]$ 的核磁共振氢谱(300 MHz, DMSO)

表 S1 离子液体 $\left[\mathrm{C}_{2} \mathrm{mim}\right][\mathrm{Ala}]$ 核磁共振氢谱数据表（300 MHz，DMSO）

\begin{tabular}{ccc}
\hline Chemical shift & Hydrogen number & Radical \\
\hline $0.982 \sim 1.017(\mathrm{t})$ & 3.0 & $\mathrm{NCH}_{2} \mathrm{CH}_{3}$ \\
$7.721(\mathrm{~d})$ & 1.0 & $\mathrm{C}(4) \mathrm{H}$ \\
$2.819 \sim 2.842(\mathrm{~m})$ & 1.0 & $\mathrm{CH}$ \\
$3.873(\mathrm{~s})$ & 3.0 & $\mathrm{NCH}_{3}$ \\
$1.372 \sim 1.428(\mathrm{~d})$ & 3.0 & $\mathrm{CHCH}_{3}$ \\
$9.692(\mathrm{~s})$ & 1.0 & $\mathrm{C}(2) \mathrm{H}$ \\
$7.815(\mathrm{~d})$ & 1.0 & $\mathrm{C}(5) \mathrm{H}$ \\
$4.162 \sim 4.241(\mathrm{t})$ & 2.0 & $\mathrm{NCH}$ \\
\hline
\end{tabular}




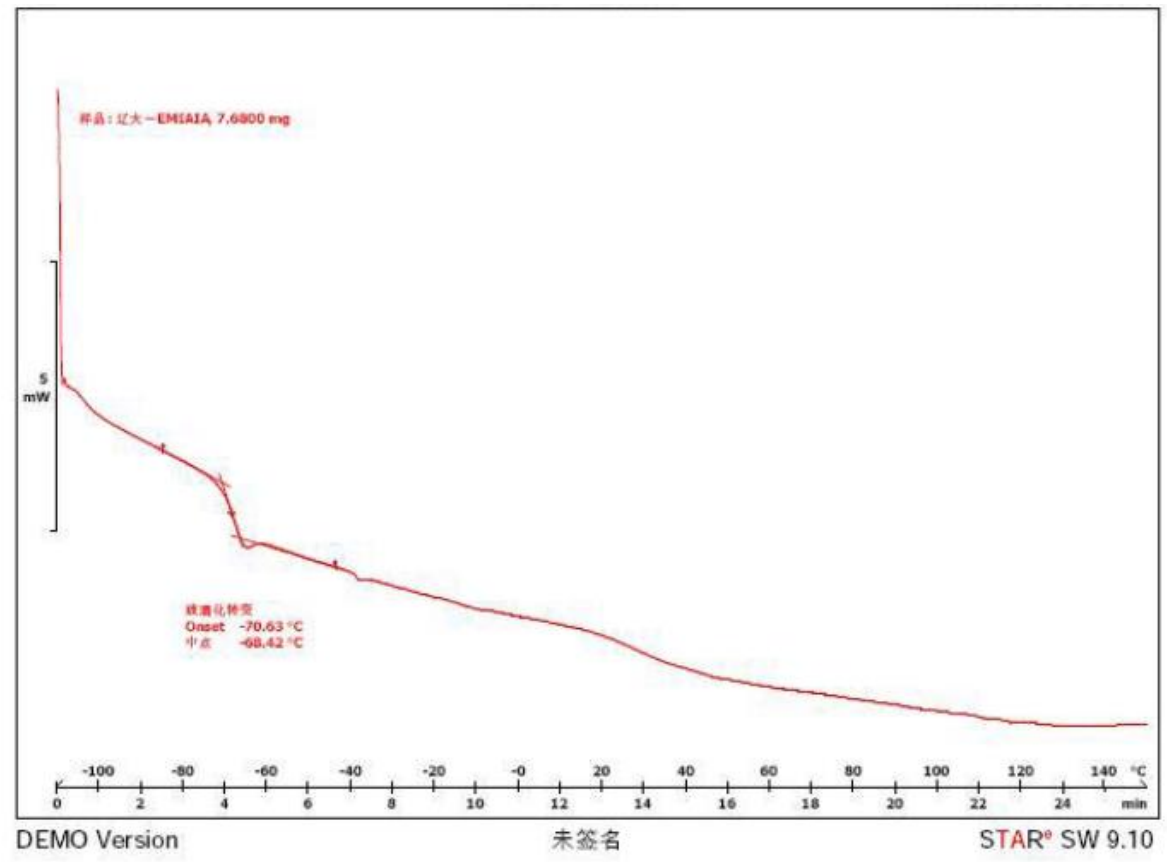

Fig.S2 离子液体 $\left[\mathrm{C}_{2} \mathrm{mim}\right][\mathrm{Ala}]$ 的 DSC 图

差式扫描量热温度的测量范围 $-110-150^{\circ} \mathrm{C}$, 加热速率为 $10^{\circ} \mathrm{C} / \mathrm{min}$, 样品测试前需在 $-110^{\circ} \mathrm{C}$ 停留使其稳定 5 分钟, 随后开始进行测试阶段。 